\title{
Photon-counting multifactor optical encryption and authentication
}

\author{
E Pérez-Cabré ${ }^{1 *}$, E A Mohammed ${ }^{2}$, M S Millán ${ }^{1}$ and H L Saadon ${ }^{2}$ \\ ${ }^{1}$ Grup d'Òptica Aplicada i Processament d'Imatges (GOAPI), Departament d'Òptica i \\ Optometria, Universitat Politècnica de Catalunya-BarcelonaTech (UPC), Violinista Vellsolà \\ 37, 08222 Terrassa (Spain) \\ ${ }^{2}$ Laser Applications and Optical Materials Laboratory, Department of Physics, College of \\ Science, University of Basrah, Basrah (Iraq) \\ *E-mail: elisabet.perez@upc.edu
}

\begin{abstract}
The multifactor optical encryption authentication method [Opt. Lett., 31, 721-3 (2006)] reinforces optical security by allowing the simultaneous authentication of up to four factors. In this work, the photon-counting imaging technique is applied to the multifactor encrypted function so that a sparse phase-only distribution is generated for the encrypted data. The integration of both techniques permits an increased capacity for signal hiding with simultaneous data reduction for better fulfilling the general requirements of protection, storage and transmission. Cryptanalysis of the proposed method is carried out in terms of chosen-plaintext and chosen-ciphertext attacks. Although the multifactor authentication process is not substantially altered by those attacks, its integration with the photon-counting imaging technique prevents from possible partial disclosure of any encrypted factor, thus increasing the security level of the overall process. Numerical experiments and results are provided and discussed.
\end{abstract}

Keywords: Optical security, multifactor encryption, photon-counting imaging, information verification, authentication, optical recognition, nonlinear correlation, Fourier optics, cryptanalysis

\section{Introduction}

Optical processing systems have been proposed for a number of security applications, including encryptiondecryption, authentication, and anti-counterfeiting of a number of primary images, from one in the most common case, up to four in the case of multifactor authentication [1-6]. They benefit from parallel processing, multiple degrees of freedom (such as amplitude, phase, wavelength, and polarization of light), high storage capacity and use of biometrics data such as, for instance, fingerprints, iris and retina.

Optical security technology is based on complex information processes in which the signals are, first, hidden from human perception or easy conversion into visible signals (to keep them secret); second, extremely difficult to reproduce with the same properties (to avoid counterfeiting); and, third, automatically, real time, robustly, and often remotely readable by compact processors that retrieve the original information and/or validate authorized signatures [4]. 
Since the pioneer work of Réfrégier and Javidi in 1995 [7], where the bases of the double random phase encoding (DRPE) were established, scientists all over the world have done considerable work in this field [26]. The experimental realization of the DRPE has been initially carried out in a $4 f$-processor [7-8], and it has been extended to the Fresnel [9-10] and Fractional Fourier [11] domains. In addition to these $4 f$-systembased optical encryption methods, the joint transform correlator (JTC) [8] has been proposed to alleviate the accurate optical alignment requirements and avoid the need of complex conjugating the key code of the $4 f$ system [12-14]. Cryptanalysis is the science that analyses the security of cipher schemes by simulating the unauthorized behaviour and resources of an opponent who wants to disclose some encrypted information. The cryptanalysis of DRPE has revealed certain vulnerability against different attacks such as chosenciphertext attack (CCA), chosen-plaintext attack (CPA) and known-plaintext attack (KPA) [15-17]. Recently, a new approach based on the integration of photon-counting imaging techniques with the DRPE has been proposed to increase the security of the encryption process with the additional achievement of a reduction of the encrypted information to be stored and transmitted [18]. The sparse encrypted distribution produces a decoded image that cannot be recognized by intruders. To verify the decrypted image, the retrieved signal is compared to the original image by applying matched filtering and a correlation-based recognition. The order of application of the two integrated techniques, photon-counting imaging and DRPE, was evaluated in a posterior work [19]. There, its effects on the authentication stage were analysed and the additional possibility of image retrieval based on the correlation results was demonstrated. From this first proposal [18] and based on the same principle of the photon-counting imaging technique, a number of contributions in the field of optical encryption algorithms have been published in the literature very recently [18-22], showing the increasing interest of this method and demonstrating its potential applicability to security systems. Even though they consider different realizations of the sparsing strategy, they commonly aim to reduce the amount of information in the encrypted distribution without substantially affecting the final verification result. It is worth mentioning that the methods proposed in Refs. [19,22] additionally reduce the sparse encrypted distribution by considering uniquely its phase information, and in particular Ref. [19] provided evidences of further reduction by using only 2 bits, or equivalently 4 phase levels, to successfully represent the relevant encrypted information for authentication purposes. 
Most of the optical security systems usually deal with a single primary image (for instance, an object, a plaintext, a signature, a biometric signal) as authenticator [2-7,10-14,18-23]. Some approaches permit to store multiple primary images, either in an optical memory [9] or in a single encrypted distribution [24-27], with the purpose of sequential and independent one-by-one decryption. Alternatively, security can be reinforced by combining several authenticators in a single encrypted distribution [1] aiming the simultaneous verification of more than one primary images. In such a case, a Boolean AND operation has to be applied to each factor's authentication results so all of them must be affirmative before the final authentication is satisfied. This principle was applied to encryption processors designed for secure authentication [1]. The multifactor optical encryption authentication (MOEA) presented in Ref. [1] describes a method to encode up to four factor authenticators in a single complex-amplitude distribution. All four factors can be different in their content and category (for instance, biometric signals, logos, patterns, plaintexts, random phase codes) [3-4,28-29]. The resulting encoded image fulfils crucial requirements for security systems: does not reveal any factor information, is extremely difficult to counterfeit and permits real-time automatic verification. MOEA was also used for near infrared (NIR) multifactor identification (ID) from remote authentication of invisible NIR ID tags [28]. The main goal of the original MOEA and its extended application to the NIR spectrum is the simultaneous verification and authentication of the multiple factors hidden in the encrypted function through correlation, and it is not primarily intended for image retrieval.

Unlike photon-counting imaging and as far as we know, the potential of MOEA has been scarcely explored in a few works [1, 28-29]. Considering their attractive properties and having in mind that both the MOEA and the photon-counting DRPE techniques have the common purpose of information authentication and verification rather than information retrieval for direct visualization, we aim to explore the potential capabilities of the combined techniques in an integrated security system. Thus, the novel integration of both, the MOEA described in Ref. [1] and the photon-counting (PhC) technique presented in [18], as it is described in this paper, is intended to provide the PhC-MOEA security system with more than just compatibility and simple addition of their respective features. Indeed, the integration should lead towards a new, more powerful, more secure and large capacity technique that would benefit from the original properties of both source techniques with further improvement: 
- Multi-factor encryption for simultaneous verification of more than one primary image (up to four primary images),

- High reduction of encrypted information to be stored and transmitted. Such a reduction becomes more significant since it would be applied to the content of more than one image and not to a single image as it is commonly the case in photon-counting based authentication approaches.

- High compression of the burden of transmitted data required for the verification and authentication stage. After photon counting would be applied in the encryption process, only the phase value with limited n-bits of the sparse pixels selected from encrypted image would be necessary for verification.

- Increased capacity for signal hiding (up to four images) with robust resistance against attacks. High protection of factor's categories against unauthorised disclosure.

- Suitability for optical implementation by means of similar setups, with non-increased alignment requirements.

In this work, the new integration of the MOEA method with the photon-counting imaging technique is detailed and numerical results are provided to show the feasibility of the proposal and the fully achievement of the improved properties just listed. Since cryptanalysis was not included in Ref. 1, where MOEA was firstly described, we will deal with it in this paper. We will show for the first time that, although the MOEA process is robust against chosen plaintext and ciphertext attacks, certain weakness related to the partial disclosure of the factor content can be found. The combination of photon-counting imaging techniques along with the multifactor verification will offer an opportunity to overcome this vulnerability. The paper briefly reviews the multifactor and photon-counting authentication techniques, and describes their integration in this new proposal in Section 2. Numerical experiments are provided in Section 3 in order to show and validate the feasibility of the novel PhC-MOEA system. Cryptanalysis of the integrated PhC-MOEA system is presented in Section 4. Finally, the main conclusions are outlined in Section 5.

\section{Photon-counting multifactor optical encryption authentication (PhC-MOEA)}

The integration of the photon-counting technique with the multifactor optical encryption is the focus of this section, which establishes the basis of the novel PhC-MOEA security system proposed in this work. We 
firstly review the main principles of both methods to provide the reader with the necessary notation and background for the following steps. Finally, the combination of both methods is detailed.

\subsection{Multifactor optical encryption authentication (MOEA)}

The multifactor optical encryption-authentication (MOEA) technique introduced in Ref. [1] was designed to validate four-factor authentication by using double random phase encoding [7], fully-phase-based encryption [30] and a combined nonlinear JTC [31] and a classical $4 f$-correlator [8]. The MOEA technique, allows the simultaneous recognition and authentication of multiple images of varied categories, with at least one of them being an independent random white sequence [28-29]. The information of the primary signals was scrambled and hidden from visual perception in the final encrypted distribution placed on a personal ID tag (or card). The reference images of the ID tag were compared with the actual input images obtained in situ for authentication.

In this section we summarize the MOEA method as it was introduced in Ref. [1] using 1D notation for the sake of simplicity. The reference primary images (biometric signals) $r(x)$ and $s(x)$ can turn out to be a noisy-like complex-valued distribution function $\psi(x)$, when two independent random white sequences $b(x)$ and $n(x)$ are used as masks to encrypt the information. All the four signals, $r(x), s(x), b(x)$, and $n(x)$, are normalized positive functions distributed in $[0,1]$. These images are phase encoded to yield $t_{r}(x), t_{s}(x)$, $t_{2 b}(x)$ and $t_{2 n}(x)$, that are generically defined by $t_{f}(x)=\exp \{i \pi f(x)\}$. The complex-amplitude encrypted function $\psi(x)$ containing the multifactor authenticators is mathematically described by

$$
\psi(x)=t_{r+2 b}(x) * t_{s}(x) * F T^{-1}\left[t_{2 n}(x)\right]
$$

where $t_{r+2 b}(x)=t_{r}(x) \cdot t_{2 b}(x)=\exp \{i \pi r(x)\} \cdot \exp \{i 2 \pi b(x)\}, F T^{-1}$ indicates inverse Fourier transform, and * the convolution operation.

In general, the encrypted function looks like a dim noisy distribution in both their magnitude and phase parts and can be reproduced on an ID card in a variety of modalities and formats [1,28-29]. 
Let us consider the second part of the process that concerns authentication. Let $p(x)$ and $q(x)$ denote the positive and normalized input images that are to be compared with the reference images $r(x)$ and $s(x)$, respectively. Let $d(x)$ and $m(x)$ be the random codes that are to be compared with $b(x)$ and $n(x)$, respectively. A possible realization of the optical processor required for the optical authentication stage combines a nonlinear JTC and a classical $4 f$-correlator (Fig. 1). In the first step, the encrypted function $\psi(x-a)$ and one phase-encoded input image, for instance, $t_{p}(x+a)=\exp \{i \pi p(x+a)\}$, are displayed side by side at a distance $2 a$ apart on the input plane of the nonlinear JTC illuminated by coherent light. In the first approach, let us assume that the random phase masks (RPMs), $t_{2 d}(x)$ and $t_{2 m}(x)$, are key phase codes known to the processor. The RPM $t_{2 d}(x+a)$ is bonded to the phase-encoded input image $t_{p}(x+a)$.

A CCD sensor placed in the Fourier plane of the JTC captures the intensity distribution $I(u)$ of the joint power spectrum:

$$
I(u)=\left|F T\left[\psi(x-a)+t_{p+2 d}(x+a)\right]\right|^{2} .
$$

The expansion of Eq. (2) gives the classical four terms, two of which convey the cross-correlation signals that lead to spatially separated distributions in the output plane [1]. These two terms can be modified based on a number of nonlinear techniques [31-32] that are useful to adjust the discrimination capability of a recognition system. The nonlinear transformation applied to the joint power spectrum of Eq. (2) is defined as

$$
N L^{k}\{I(u)\}=I(u) \cdot|I(u)|^{k-1},
$$

where the parameter $k$ defines the strength of the applied nonlinearity. In a $k$ th-law processor, a linear filtering technique is obtained for $k=1$, whereas $k=0$ leads to a phase extractor that generally enhances the high frequency content. The resultant nonlinearly modified joint power spectrum is displayed on the Fourier plane of a $4 f$-classical correlator (Fig. 1), where, at the same time, the phase-encoded input image $t_{q}(x)$ is introduced in the correlator input plane and $t_{2 m}(x)$ in the Fourier plane. The interesting term obtained just behind the Fourier plane is

$$
\left[T_{q}(u) T_{s}^{*}(u)\left|T_{s}(u)\right|^{k-1}\right]\left[T_{r+2 b}^{*}(u) T_{p+2 d}(u)\left|T_{r+2 b}(u) T_{p+2 d}(u)\right|^{k-1}\right]\left[t_{2 n}^{*}(u) t_{2 m}(u)\right] \exp \{i 2 \pi(2 a) u\},
$$


where a function in uppercase letter indicates the Fourier transform of the function in lowercase letter and $u$ is the spatial frequency coordinate. If the AND condition $r(x)=p(x), s(x)=q(x), b(x)=d(x)$ and $n(x)=m(x)$ is fulfilled and $k=0$, then the term of Eq. (4) focuses on a sharp multifactor autocorrelation (AC) peak, spatially separated from other terms and centred at $x=2 a$ corresponding to the cross-correlation of the AC signals given by

$$
\left|A C_{P O F}\left[t_{s}(x)\right] \otimes A C_{P P C}^{*}\left[t_{r+2 b}(x)\right] \otimes A C_{C M F}^{*}\left[T_{2 n}(x)\right] * \delta(x-2 a)\right|^{2} .
$$

Symbol $\otimes$ in Eq. (5) denotes cross correlation and subindexes CMF (classical matched filter), POF (phaseonly filter), and PPC (pure phase correlation) indicate the type of filter involved in the AC signal $[1,33]$. Consequently, the information contained in Eq. (4) allows reinforced security verification by simultaneous multifactor authentication. If any of the four input factors $(p(x), q(x), d(x), m(x))$ is different from the corresponding reference primary image $(r(x), s(x), b(x), n(x))$ stored in the ID tag or the system data base, then Eq. (4) contains a cross-correlation signal that is, in general, broader and less intense than the multifactor AC peak of Eq. (5) [1,28-29].

In a more general approach, the RPMs represented by $t_{2 b}(x)$ and $t_{2 n}(u)$ can be used as additional authenticators. Therefore, up to four factors can be authenticated by comparing the set of four primary images contained in the encrypted function with the set of four input images in pairs [3-4,28-29].

\subsection{Photon-counting imaging technique}

A photon-counting limited real-valued image, $f_{p h}(x)$, can be generated by controlling the expected number of incident photons in the entire scene, $N_{p}$. The probability of counting $l_{j}$ photons at pixel $x_{j}, p_{d}\left(l_{j} ; \alpha_{j}\right)$, can be shown to be Poisson distributed [34-36]:

$$
p_{d}\left(l_{j} ; \alpha_{j}\right)=\frac{\left(\alpha_{j}\right)^{l_{j}} e^{-\alpha_{j}}}{l_{j} !}, \quad l_{j}=0,1,2, \ldots
$$


where $l_{j}$ is the number of photons detected at pixel $x_{j}$ and the Poisson parameter, $\alpha_{j}$, is given by $\alpha_{j}=N_{p} g\left(x_{j}\right)$ with $g\left(x_{j}\right)$ being the normalized irradiance at pixel $x_{j}$, such that $\sum_{j=1}^{M} g\left(x_{j}\right)=1$, and $M$ equals the total number of pixels in the scene.

The photon-counting imaging approach can also be applied to the complex-valued encrypted distribution $\psi(x)$ of Eq. (1)). Let us do it in the following way: The photon-counting imaging operation is firstly applied to the real-valued magnitude distribution, $|\psi(x)|$, of the encrypted function. The photon-limited magnitude encrypted distribution, $\left|\psi_{p h}(x)\right|$, can be generated from the normalized magnitude distribution $\left|\psi\left(x_{j}\right)\right| / \sum_{j=1}^{M}\left|\psi\left(x_{j}\right)\right|$ using Equation (6). The sparse pixels $x_{i}$ selected by this operation, that is, those with non-zero photons $\left(l_{i} \neq 0\right)$, are also those whose phase value $\varphi_{\psi}\left(x_{i} ; n\right)$ will be taken with $n$-bit resolution to generate a phase-only photon-limited encrypted function $\psi_{p h}(x)$. Using a mathematical expression,

$$
\psi_{p h}(x)=\left\{\begin{array}{cc}
\varphi_{\psi}\left(x_{i} ; n\right), & l_{i} \neq 0 \\
0, & \text { otherwise }
\end{array} .\right.
$$

The DRPE [7] has been combined with photon-counting imaging or other sparsing algorithms [18-20,22] with satisfactory authentication results. Typically, the phase information is distributed from 0 to $2 \pi$ with a resolution of $n=8$ bits. But, as demonstrated in Ref. [19], further compression of information can be successfully applied, up to $n=2$ bits, with no significant loss of discrimination ratio in the correlation-based verification. Up to now, however, these algorithms have been applied only to the authentication of a single primary image hidden from direct human perception.

\subsection{Photon-counting multifactor optical encryption authentication (PhC-MOEA)}

A novel security system is proposed by applying the photon-counting imaging technique to the multifactor optical encryption authentication method, which results in the PhC-MOEA system. The most promising feature of this system is its capability of simultaneous authentication of up to four factors, giving the $\mathrm{PhC}$ MOEA system a higher degree of security than previous proposals [18-20,22]. Other relevant properties 
concerning reduction of the encrypted information to be stored and transmitted as well as the feasibility for optical implementation are to be studied in subsequent sections.

Four factors, $r(x), s(x), b(x)$ and $n(x)$, with no a priori constrain, are appropriately merged into a multifactor encrypted distribution, $\psi(x)$, which is in general of a complex-valued nature. From it, a sparse and phase-only encrypted function is obtained by applying the photon-counting imaging technique described above, $\psi_{p h}(x)$ for a given number of photon-counts $N_{p}$ (Eq. (7) and inset of Fig.1). To validate the information of the four factors, we compute the output intensity distribution of the PhC-MOEA with phase extraction nonlinearity, $k=0$ in Eq. (4). A positive validation is obtained only when all four factor's authentication results are correct. Thus, the output intensity distribution will correspond to a high and sharp multifactor autocorrelation peak. Any mismatch between the four signals and the information included in the encrypted function, will lead to an important decrease of the output peak, just indicating a situation of rejection or non positive result (e.g. denied permission, verification not possible, false information). It is worth remarking the suitability of PhC-MOEA for optical implementation by means of a setup similar to that used for MOEA, with non-increased alignment requirements, as it is sketched in Fig. 1.

\section{PhC-MOEA through numerical experiments}

The PhC-MOEA procedure is illustrated here with the following numerical experiment. Let us consider the biometric retina images $(188 \times 188$ pixels $)$ as primary reference images $r(x)$ and $s(x)$ along with the phase masks generated by two random white sequences $b(x)$ and $n(x)$ (Fig. 2 and Refs. [1,4]). The encrypted distribution $\psi(x)$, shown if Fig. 3(a), is obtained using Eq. (1) and, from it, a phase-only photon-limited version of the encrypted function is computed $\psi_{p h}(x)$, with a number of photon counts $N_{p}=10^{3}$ and $n=8$ bit resolution. The chosen value $N_{p}=10^{3}$ corresponds to $2.8 \%$ of the image size. Figure $3(\mathrm{~b})$ shows the pixels whose phase value has been used to generate $\psi_{p h}(x)$ in this numerical experiment. To validate the information of the four factors simultaneously, we compute the output intensity distribution of the PhC- 
MOEA with $k=0$ (Eq. (4)). When all four factor's authentication results are correct, that is, $p(x)=r(x)$, $q(x)=s(x), d(x)=b(x)$ and $m(x)=n(x)$, the normalized output intensity distribution is plotted in Figure 3(c). The obtained output shows a high and sharp multifactor autocorrelation peak that accounts for a final positive authentication, despite the drastic reduction of information produced by the photon-counting technique.

Any mismatch between the four signals $r(x), s(x), b(x)$ and $n(x)$ scrambled and hidden in the phase-only photon-limited encrypted function, $\psi_{p h}(x)$, and the second set of four signals consisting of $p(x), q(x)$, $d(x)$ and $m(x)$, leads to an important decrease of the output correlation peak (Table 1). For instance, Figure 3(d) shows the output distribution when another person, different from the authorized user, is analysed by the processor, that is, when $r(x) \neq p(x), s(x) \neq q(x)$ (being $p(x)$ and $q(x)$ the images of Fig.33.10, Pair 2, in Ref. [4]), but still $b(x)=d(x)$ and $n(x)=m(x)$. All the other analysed situations included in Table 1 obtain a similar output as the one depicted in Fig. 3(d).

These results demonstrate the feasibility of the integration of the photon counting imaging technique along with the multifactor optical encryption authentication (PhC-MOEA). Apart from the satisfactory multifactor authentication results shown in Fig. 3 and Table 1 that prove a powerful, highly secure, and large capacity verification system, the combined application of PhC-MOEA technique provides the additional advantage of data compression, which is an important feature for information storage and transmission in the context of the whole security process.

To establish the most convenient nonlinearity (value of parameter $k$ ) and the most suitable number of photons (parameter $N_{p}$ ) that give the best performance of the proposed PhC-MOEA system, two metrics are going to be used: the $D R$ (discrimination ratio) and $P C E$ (peak-to-correlation energy). Both metrics are widely known parameters used to evaluate the performance of pattern recognition systems based on optical correlation [37-38].

The discrimination ratio $(D R)$ metric is related to the ratio between the maximum peak value of the crosscorrelation output, $C C$, and the maximum peak value of autocorrelation of the reference target, $A C$, [38] 


$$
D R=\left|1-\frac{C C}{A C}\right|
$$

It provides information about the capacity of the recognition system for discerning small differences between objects.

The peak-to-correlation energy $(P C E)$ parameter, defined as the ratio between the maximum intensity peak value and the total energy of the output plane [37],

$$
P C E=\frac{\text { correlation peak energy }}{\text { correlation plane energy }}
$$

is usually a good indicator of the sharpness and height of the output correlation peak.

The performance of the proposed security system in terms of $D R$ and $P C E$ has been analysed from the results obtained from 20 numerical experiments where the retinal scans, $r(x), s(x), p(x)$ and $q(x)$, as well as the key codes, $n(x)$ and $b(x)$, were kept unchanged, while the random process involved in the Poisson distribution (Eq. (6)) was sequentially repeated with different parameter values during the photon-counting imaging stage. Figure 4 depicts the mean $D R$ value computed from the set of numerical simulations versus the number of photon counts $\left(N_{p}\right)$ with various $k$ values. The standard deviations of the numerical simulations permit us to estimate the uncertainties. If we establish an arbitrary threshold of $D R=0.5$, we remark that $D R$ values obtained for nonlinearities $k \in[0,0.5]$ and a number of photon counts of $N_{p}=500$ or higher, good discrimination ratios $(D R>0.5)$ are achieved. In particular, for $N_{p}=10^{3}$ (or equivalently $2.8 \%$ of the image pixels) and $k=0$, the $D R$ is above 0.8 .

For small numbers of $N_{p}$ (lower than 500), the $D R$ decreases rapidly for all tested $k$ values, reaching similar mean $D R$ values for the different applied nonlinearities, and with increased uncertainties. Such behaviour can be likely due to the small number of pixels that convey the information in the encrypted distribution.

In general, small values of $k$ along with $N_{p}$ values higher than 500, provide satisfactory results in terms of $D R$. In such a situation, the verification system presents a good discrimination capability when the integration of PhC-MOEA is considered. 
Figure 5 plots the mean $P C E$ values versus $N_{p}$ for the set of 20 numerical experiments described above. A nearly linear performance appears in the graphs for all tested $k$ values. $P C E$ increases for increasing $N_{p}$ photon-counts. Among the considered nonlinearities, $k=0$ provides the highest $P C E$ values for the range of $N_{p}$ values considered.

According to the results depicted in Fig. 4 and Fig. 5, a number of photons of $N_{p}=10^{3}$ and $k=0$ are chosen for the numerical experiments carried out to show the feasibility of the proposal.

\section{Cryptanalysis of the MOEA and the PhC-MOEA systems}

In the previous Section, we have shown the feasibility of the novel PhC-MOEA integration in order to achieve satisfactory simultaneous multifactor authentication. In this Section we point out the utility of such integration from the point of view of security. To do so, we are going to test the resistance of the standard MOEA [1] and the newly proposed PhC-MOEA systems against two different types of attacks: chosenplaintext attacks (CPA) and chosen-ciphertext attacks (CCA).

Chosen-plaintext attacks (CPA) [39] can be defined as a situation where the attacker has access to probe the encryption machine to encrypt specific plaintexts in order to obtain the corresponding ciphertexts with the goal of extracting further information, mainly the secret key. In a chosen ciphertext attack (CCA), the adversary has a chance to enter one or more chiphertexts into the decryption machine and obtain the corresponding plaintexts [15]. From these pieces of information the attacker may recover crucial information of the security system such as the hidden decryption key. For the interested reader, the security flaws of the DRPE are analysed elsewhere ([15-17] for a $4 f$-processor, [39-40,13-14] for the JTC architecture).

\subsection{Cryptanalysis of the MOEA}

First, we analyse the security level of MOEA method [1] against CPA and CCA. Figure 2 shows the four signals used in the numerical experiments, two pieces of biometrics and two random codes to generate the RPMs. The obtained results for this cryptanalysis will be compared to the positive verification of four factors without alteration of the encryption-decryption scheme shown in Fig. $3 \mathrm{~b}$ and Table 1. 
Let us suppose that an unauthorized user has access to the encryption stage in an analogous way to CPA. In such a situation the intruder may be able to counterfeit the encrypted distribution with the purpose of achieving a positive authentication when going through the established decryption and verification step. As chosen-plaintext attacks, the intruder can use different plaintexts. Let us consider two common plaintexts such as a dot function

$$
w_{1}(x)=\operatorname{dot}(x)=\left\{\begin{array}{ll}
1 & \text { for } x=0, \\
0 & \text { elsewhere }
\end{array},\right.
$$

and a zero uniform input image

$$
w_{2}(x)=0,
$$

being $w_{i}(x)$ with $i=1,2$, the images used by the intruder to substitute either $r(x)$ or $s(x)$. Different combinations of the input plaintexts are tested in the cryptanalysis (Table 2). In these experiments, the two random phase codes, $b(x)$ and $n(x)$, are supposed to be known by the processor. The encrypted distribution is obtained as defined by Eq. (1) and in all the cases the resulting ciphertexts keep their noisy appearance without revealing the four-signal content. As an example, Figure 6 shows the amplitude and phase distributions of the encrypted function corresponding to $r(x)=\operatorname{dot}(x)$ and $s(x)=\operatorname{dot}(x)$.

The resulting ciphertexts are then introduced in the decryption-verification stage, as it was described in Section 2.1, with the attacker aiming a positive authentication. The verification stage requires the comparison of the four factors hidden in the ciphertext $(r(x), s(x), b(x), n(x))$ with the attacker retinal scans captured in situ from the non-authorized both eye fundus $(p(x), q(x))$ and the RPMs obtained from the processor database $(d(x), m(x))$. To illustrate the MOEA performance, Figure 7 depicts the output results for several situations: Fig. 7(a) contains a positive validation (unaltered ciphertext, $p(x)=r(x), q(x)=s(x)$ and correct RPMs $d(x)=b(x)$ and $m(x)=n(x)[1,4])$ to facilitate the comparison with the cryptanalysis results; Fig. 7(b) corresponds to a counterfeit ciphertext with $r(x)=\operatorname{dot}(x)$ and $s(x)=0$; and Fig. 7(c) with $r(x)=\operatorname{dot}(x)$ and $s(x)=\operatorname{dot}(x)$, both of them compared to the in situ captured retinal images $p(x)=r(x)$ and $q(x)=s(x)$ and the phase codes $d(x)=b(x)$ and $m(x)=n(x)$ from the database. All the results are 
shown in 2D image format and also in 3D representation in Fig. 7. They all correspond to the applied nonlinearity $k=0$. A summary of the whole cryptanalysis results for this experiment is provided in Table 2 . For all the evaluated situations, only the positive validation achieves a high and sharp correlation peak (Fig. 7(a)) that can be distinguished without any doubt from the low noisy energy outputs obtained in the altered procedures. Thus, as a first conclusion, it is highly remarkable that the authentication results for all the manipulated ciphertexts are always negative, which indicates a robust behaviour of the MOEA scheme against the described CPAs.

However, if we further look at the obtained output planes, we can distinguish between two cases. On the one hand, if the input signal $s(x)$ is replaced by a uniform zero image $(s(x)=0)$ in the altered ciphertext, the output plane consists of a low noisy background without any remarkable information on it, independently of the signal assigned to the input image $r(x)$ (Fig. 7b). Such a situation rejects the counterfeit encrypted function. On the other hand, if function $s(x)$ is replaced by a dot function $(s(x)=\operatorname{dot}(x))$, the output plane will partially reveal the biometric signal used as function $q(x)$ with a noisy appearance after the decryption stage (Fig. 7c). As the retina scan, captured in situ from the person willing access to the system, is displayed on the background of the verification plane, the attacker can recognize at least the nature of the information used for encryption and verification. This disclosure can be considered a weakness of the system.

We also explore the resistance of the standard MOEA method [1] to CCA. In a chosen-ciphertext attack, an unauthorized user has access to the decryption machine (verification stage). Let us imagine that the intruder is able to obtain a valid encrypted function with the information of four correct factors. The aim of the attacker is to achieve a positive multifactor validation by altering signals $p(x)$ and $q(x)$ in the decryptionverification stage. In the CCA analysis, we consider several combinations of manipulated functions $w_{1}(x)$ and $w_{2}(x)$ (Eqs. (10) and (11)), where $w_{i}(x)$ with $i=1,2$, can substitute either functions $p(x)$ or $q(x)$. Table 3 summarizes the obtained results for all the cases evaluated in this experiment. Figure 8(a) shows the output verification plane for $p(x)=\operatorname{dot}(x), q(x)=0$ and the correct RPMs $(d(x)=b(x)$ and $m(x)=n(x))$ from the processor database. The applied nonlinearity corresponds to $k=0$. A uniform low- 
energy output is obtained, which indicates a rejection of the non-authorized signals. When $q(x)=0$, independently of the value of function $p(x)$ a similar result is achieved. If function $q(x)$ is set to zero (the input $t_{q}(x)$ is a uniform image), its Fourier transform is a Dirac delta function that multiplies the distribution inserted in the Fourier plane of the $4 f$-correlator in Fig. 1. The output plane of the verification processor will then be a constant uniform value as it is displayed in Fig. 8(a).

Figure $8(\mathrm{~b})$ depicts the output plane for $p(x)=\operatorname{dot}(x), q(x)=\operatorname{dot}(x)$ and the correct RPMs $(d(x)=b(x)$ and $m(x)=n(x))$ from the processor database. A negative authentication is also achieved as a result of the low energy output obtained in this situation. It is remarkable that none false authentications have arisen in the multifactor verification procedure when CCA are tested. However, a dim reproduction of the retinal scan corresponding to function $s(x)$ is revealed on the background of the output plane when $q(x)=\operatorname{dot}(x)$, independently of the value of function $p(x)$. Again, some information disclosure is unacceptably obtained when some CCA are directed against the MOEA system.

Even though the authentication outcome is not seriously affected by the chosen-text attacks (both CPA and CCA), the partial disclosure of confidential information is unacceptable. For this reason, the new PhCMOEA method is proposed in this paper to increase the security of this multifactor optical encryption authentication procedure.

\subsection{Cryptanalysis of the PhC-MOEA system}

Firstly, let us suppose that the attacker has access to the encryption machine so that different combinations of chosen input images are introduced instead of functions $r(x)$ and $s(x)(\mathrm{CPA})$. The phase codes $n(x)$ and $b(x)$ are correctly and automatically introduced from the system database. The attacker considers dot functions or uniform zero functions (Eqs. (10) and (11)) as input images. The altered photon-counting encrypted distribution generated from such a procedure is afterwards tested by comparing the embedded multifactor information with the in situ captured retinal images $(p(x), q(x))$ and the correct key codes provided by the database $(d(x), m(x))$. A summary of the numerical results for $k=0, N_{p}=10^{3}$ and $n=8$ 
bits is provided in Table 4 and two examples of the output plane obtained from the authentication system are depicted in Figs. 9(a)-(b). In all tested cases, the authentication system does not validate the multifactor information since a low noisy background without any sharp intensity peak is obtained. Moreover, unlikely the case of the former MOEA system where one retina image was partially disclosed (when $r(x)=\operatorname{dot}(x)$ and $s(x)=\operatorname{dot}(x)$, see Fig. 7(c)), the dim appearance of the PhC-MOEA output plane does not reveal any information about the primary signals hidden in the encrypted distribution (Fig. 9, in particular when $r(x)=\operatorname{dot}(x)$ and $s(x)=\operatorname{dot}(x)$ in Fig. 9(b)). In that sense, the PhC-MOEA technique achieves a superior degree of security in comparison to the former MOEA system.

To further investigate the cryptanalysis of PhC-MOEA, we analyse now the possibility that an attacker manipulates the decryption and authentication machine (CCA). Let us suppose that the intruder has access to the verification stage and chooses two different images, $w_{1}(x)=\operatorname{dot}(x)$ and $w_{2}(x)=0$ (Eqs. (10)-(11)), to tamper the process. Different input image combinations have been explored and the obtained results are summarized in Table 5. In all cases, parameters $k=0, N_{p}=10^{3}$ and $n=8$ bits have been set to carry out the authentication procedure. Figures $10(\mathrm{a})$ and $10(\mathrm{~b})$ depict the output planes corresponding to two representative cases among the analysed possibilities. Figure 10(a) corresponds to the case where $p(x)=\operatorname{dot}(x)$ and $q(x)=0$, while Fig. 10(b) shows the output result for $p(x)=\operatorname{dot}(x)$ and $q(x)=\operatorname{dot}(x)$, so that they can be considered as the analogous cases to the single MOEA procedure, aforementioned in Section 4.1 and displayed on Figs. 8(a)-(b), respectively. Numerical simulations confirm that the whole set of the analysed CCA attacks conduct similar authentication results to the output planes shown in Figs. 10(a) and 10(b).

We remark that now, no confidential information is disclosed. Additionally, the output planes consist of a very low background without any intensity peak, which indicates that the multifactor information is not validated for any of the considered attacks.

In particular, for $q(x)=0$, the output plane consists of a constant value (Fig. 10(a)), as it corresponds to the Fourier transform of a modulated Dirac delta distribution (see Section 4.1). For the case of $q(x)=\operatorname{dot}(x)$, the output plane corresponds to a low noisy background (Fig. 10(b)) that, differently to Fig. 8(b), does not 
reveal the information of any factor embedded in the encrypted distribution, in particular the eye fundus image.

Finally, we conclude that for the analysed CCA, the PhC-MOEA technique has an improved performance in comparison with the prior MOEA system. In all the analysed cases, the photon-counting imaging technique permits to increase the security of the multifactor optical verification system.

\section{Conclusions}

In this work, a new multifactor optical encryption-authentication system combined with the photon-counting imaging technique is proposed. Not only have the properties of the respective source techniques been made compatible and preserved but also the dubbed photon-counting multifactor optical encryption authentication (PhC-MOEA) system proves to be a higher level security system, more powerful and secure than the original versions (MOEA [1] and Photon-counting DRPE [18] authentication systems). Among the main properties achieved by the integrated PhC-MOEA system it is worth remarking data reduction and compression. This is based on the facts that, first, up to four primary images can be scrambled into a single encrypted image and simultaneously verified, and second, the sparse encrypted function obtained after applying photon-counting is additionally made phase-only and can be lowered to $n$-bit grey level resolution (2 bits can suffice [19]). The integration also achieves an additional protection of factor's categories against unauthorised attacks, and finally the same MOEA setup is suitable to implement the novel PhC-MOEA method without increasing the system complexity.

Conducted numerical experiments have allowed us to establish the ranges of two parameters, the number of photon counts $\left(N_{p}\right)$ and the severity of the nonlinerarity in the authentication stage $(k)$, so as to determine the best system performance in terms of $D R$ and $P C E$ values.

The cryptanalysis proves that the partial disclosure of confidential information shown by the MOEA system under certain attacks is successfully overcome by the PhC-MOEA system. 


\section{Acknowledgements}

M. S. Millán and E. Pérez-Cabré thank the Spanish Ministerio de Ciencia e Innovación and Fondos FEDER for financial support (project number DPI2013-43220-R). E. A. Mohammed and H. L. Saadon thank the support of this work by Iraqi Ministry of Higher Education and Scientific Research (Grant No. 2013/15578). 


\section{References}

[1] Millán M S, Pérez-Cabré E and Javidi B 2006 Multifactor authentication reinforces optical security Opt. Lett. 31 721-3

[2] Javidi B (ed) 2005 Optical and Digital Techniques for Information Security (Berlin: Springer)

[3] Matoba M, Nomura T, Pérez-Cabré E, Millán M S and B. Javidi 2009 Optical techniques for information security, Proc. IEEE 97 1128-48

[4] Millán M S and Pérez-Cabré E 2011 Optical data encryption in Optical and Digital Image Processing: Fundamentals and Applications (Eds. G. Cristóbal, P. Schelkens and H. Thienpont), Wiley-VCH Verlag GmbH \& Co 739-67

[5] Liu S, Guo C and Sheridan J T 2014 A review of optical image encryption techniques Optics \& Laser Technology 57 327-42

[6] Chen W, Javidi B and Chen X 2014 Advances in optical security systems Advances in Optics \& Photonics 6 120-55

[7] Réfrégier P and Javidi B 1995 Optical image encryption based on input plane and Fourier plane random encoding Opt. Lett. $20767-9$

[8] Goodman J W 1996 Introduction to Fourier Optics (Second edition, McGraw-Hill, New York)

[9] Matoba O and Javidi B 1999 Encrypted optical memory system using three-dimensional keys in the Fresnel domain Opt. Lett. 24 762-4

[10] Situ G and Zhang J 2004 Double random-phase encoding in the Fresnel domain Opt. Lett. 29 1584-6

[11] Unnikrishnan G, Joseph J and Singh K 2000 Optical encryption by double-random phase encoding in the fractional Fourier domain Opt. Lett. 25 887-9

[12] Nomura T and Javidi B 2000 Optical encryption using a joint transform correlator architecture Opt. Eng. 39 2031-5

[13] Vilardy J, Millán M S and Pérez-Cabré E 2013 Improved decryption quality and security of a joint transform correlator-based encryption system Journal of Optics 15025401

[14] Vilardy J, Millán M S and Pérez-Cabré E 2014 Nonlinear optical security system based on a joint transform correlator in the Fresnel domain Applied Optics 53 1674-82 
[15] Carnicer A, Montes-Usategui M, Arcos S and Juvells I 2005 Vulnerability to chosen-cyphertext attacks of optical encryption schemes based on double random phase keys Opt. Lett. 30 1644-6

[16] Frauel Y, Castro A, Naughton T J and Javidi B 2007 Resistance of the double random phase encryption against various attacks Opt. Express. 15 10253-65

[17] Situ G, Pedrini G and Osten W 2010 Strategy for cryptanalysis of optical encryption in the Fresnel domain Applied Optics $\mathbf{4 9}$ 457-62

[18] Pérez-Cabré E, Cho M and Javidi B 2011 Information authentication using photon-counting doublerandom phase encrypted images Opt. Lett. 36 22-4

[19] Pérez-Cabré E, Abril H, Millán M S and Javidi B 2012 Photon-counting double-random-phase encoding for secure image verification and retrieval Journal of Optics 14094001

[20] Chen W, Chen X, Stern A and Javidi B 2013 Phase-modulated optical system with sparse representation for information encoding and authentication IEEE Photonics Journal 56900113

[21] Chen W and Chen X 2014 Optical color-image verification using multiple-pinhole phase retrieval Journal of Optics 16075403

[22] Chen W and Chen X 2014 Double random phase encoding using phase reservation and compression Journal of Optics 16025402

[23] Rajput S K and Nishchal N K 2012 Image encryption and authentication verification using fractional nonconventional joint transform correlator Optics and Lasers in Engineering 50 1474-83

[24] Tao R, Xin Y and Wang Y 2007 Double image encryption based on random phase encoding in the fractional Fourier domain Optics Express 15 16067-79

[25] Liu Z and Liu S 2007 Double image encryption based on iterative fractional Fourier transform Optics Communications 275 324-9

[26] Liu Z Chen H Liu T et al 2010 Double-image encryption based on the affine transform and the gyrator transform J Opt 12035407

[27] Rajput S K and Nishchal N K 2014 An optical encryption and authentication scheme using asymmetric keys Journal of the Optical Society A 31 1233-8

[28] Pérez-Cabré E, Millán M S and Javidi B 2007 Near infrared multifactor identification tags Opt. Exp. 15 $15615-27$ 
[29] Millán M S, Pérez-Cabré E and Javidi B 2006 High secure authentication by optical multifactor ID tags Proc. SPIE 6394 63940J

[30] Towghi N and Javidi B 1999 Fully phase encrypted image processor JOSA A 16 1915-27

[31] Javidi B 1989 Nonlinear joint power spectrum based optical correlation Applied Optics 28 2358-67

[32] Pérez-Cabré E, Chalasinska-Macukow K, Styczynski K, Kotynski R, Millán M S 1997 Dual nonlinear correlator based on computer controlled joint transform processor: digital analysis and optical results J. Mod. Opt. $441535-52$

[33] Pérez-Cabré E, Millán M S and Chalasinska-Macukow K, 1998 Dual nonlinear correlator applied to textured and colour object recognition Proc. SPIE 3409 444-55

[34] Yeom S, Javidi B and Watson E 2005 Photon counting passive 3D image sensing for automatic target recognition Opt. Express 13 9310-30

[35] Cho M, Mahalanobis A and Javidi B 2011 3D passive photon counting automatic target recognition using advanced correlation filters Opt. Lett 36 861-3

[36] Goodman J W 2000 Statistical Optics (New York: Wiley)

[37] Vijaya Kumar B V K and Hassebrook L 1990, Performance measures for correlation filters Appl. Opt. 29 2997-3006

[38] Millán M S, Pérez-Cabré E and Chalasinska-Macukow K 1999 Pattern recognition with variable discrimination capability by dual non-linear optical correlation Opt. Commun. 161 115-22

[39] Lin C 2012 Analysis and design of attack free generalized joint transform correlator optical encryption scheme Opt \& Las Tech 44 2032-36

[40] Barrera J F, Vargas C, Tebaldi M and Torroba R 2010 Chosen-plaintext attack on a joint transform correlator encrypting system Opt. Commun. 283 3917-21 


\section{List of Figure captions}

Figure 1. Optical processor for the MOEA system. The inset shows how it can be straightforwardly adapted to implement PhC-MOEA authentication.

Figure 2. Four factors used in the numerical experiments [1,4]: Retina images $(r(x)$ and $s(x))$ and key codes $(b(x)$ and $n(x))$ to generate the RPMs. All images are 188x188 pixel size.

Figure 3. (a) Magnitude of the multifactor encrypted function $\psi(x)$ containing the four factors shown in Fig. 2. (b) Pixels with non-zero magnitude of the photon-limited encrypted distribution, $\left|\psi_{p h}(x)\right|$, with $N_{p}=10^{3}$. (c) Output correlation intensity distribution when $p(x)=r(x), q(x)=s(x), d(x)=b(x)$ and $m(x)=n(x)$. (d) Output correlation intensity distribution when a non-authorized person is analysed by the processor, with $p(x) \neq r(x)$ and $q(x) \neq s(x)$ (taken from Fig. 33.10 of Ref. [4]), but still $d(x)=b(x)$ and $m(x)=n(x)$. Correlation intensities are normalized to the maximum peak value corresponding to the positive validation (b).

Figure 4. $D R$ versus $N_{p}$ for the PhC-MOEA system with different applied nonlinearities ( $k$ value). The represented values along with their uncertainty bars are established by running 20 numerical experiments of the random process of Poisson distribution in the photon-counting technique and taking the mean value and the standard deviation, respectively.

Figure 5. $P C E$ versus $N_{p}$ for the PhC-MOEA system with different applied nonlinearities ( $k$ value). Values and their uncertainties are computed as in Fig. 4.

Figure 6. Magnitude, $|\psi(x)|$, and phase distribution, $\varphi_{\psi}(x)$, of the altered encrypted function $\psi(x)$ obtained with the MOEA procedure when a CPA is produced in the encryption stage $(r(x)=\operatorname{dot}(x)$ and $s(x)=\operatorname{dot}(x))$.

Figure 7. 2D and 3D representation of the output authentication planes: (a) correct four factor authentication when $p(x)=r(x), q(x)=s(x)$; (b) negative authentication for CPA with $r(x)=\operatorname{dot}(x)$ and $s(x)=0$; 
and (c) negative authentication for CPA with $r(x)=\operatorname{dot}(x)$ and $s(x)=\operatorname{dot}(x)$. In all cases, RPMs $b(x)$ and $n(x)$ are correctly provided from the system database and the nonlinearity $k=0$ is applied. Maximum intensity peaks are normalized to the correct four-factor authentication case shown in (a).

Figure 8. $2 \mathrm{D}$ and $3 \mathrm{D}$ representation of the output authentication planes: negative authentication for CCA with (a) $p(x)=\operatorname{dot}(x)$ and $q(x)=0$ and (b) $p(x)=\operatorname{dot}(x)$ and $q(x)=\operatorname{dot}(x)$. In all cases, RPMs $b(x)$ and $n(x)$ are correctly provided from the system database and the nonlinearity $k=0$ is applied. Maximum intensity peaks are normalized to the correct four-factor authentication case shown in Fig. 7(a).

Figure 9. 2D and 3D representation of the output authentication planes for the PhC-MOEA system: Negative authentication for CPAs with (a) $r(x)=\operatorname{dot}(x)$ and $s(x)=0$ and (b) $r(x)=\operatorname{dot}(x)$ and $s(x)=\operatorname{dot}(x)$. In both cases, RPMs $b(x)$ and $n(x)$ are correctly provided from the system database, the nonlinearity $k=0$ is applied, $N_{p}=10^{3}$, and phase values in the photon-limited encrypted function are stored with 8-bit resolution. Maximum intensity peaks are normalized to the correct four-factor authentication case shown in Fig. 3(c).

Figure 10. $2 \mathrm{D}$ and $3 \mathrm{D}$ representation of the output authentication planes for the PhC-MOEA system: Negative authentication for CCAs with (a) $p(x)=\operatorname{dot}(x)$ and $q(x)=0$ and (b) $r(x)=\operatorname{dot}(x)$ and $q(x)=\operatorname{dot}(x)$. In both cases, RPMs $b(x)$ and $n(x)$ are correctly provided from the system database, the nonlinearity $k=0$ is applied, $N_{p}=10^{3}$, and phase values in the photon-limited encrypted function are stored with 8-bit resolution. Maximum intensity peaks are normalized to the correct four-factor authentication case shown in Fig. 3(c). 


\section{List of Table captions}

Table 1. Numerical experiments to validate the proposal of PhC-MOEA technique. Values of $k=0$, $n=8$ bits, and $N_{p}=10^{3}$ are chosen for the encryption-authentication procedure. Maximum peak values are referred to the normalized maximum peak value corresponding to the case of positive four-factor validation (Fig. 3(c)).

Table 2. Numerical experiments simulating an intruder having access to the MOEA encryption machine (CPA). Several combinations of altered input images are considered as CPAs. Authentication results are normalized to the positive four-factor verification (Fig. 7(a) and Refs. [1,4]) for the sake of comparison. All cases correspond to the applied nonlinearity $k=0$ and RPMs $b(x)$ and $n(x)$ are correctly provided by the system database.

Table 3. Numerical experiments simulating an intruder having access to the MOEA decryptionauthentication machine (CCA). Several combinations of altered input images are considered as CCAs. Authentication results are normalized to the positive four-factor verification (Fig. 7(a) and Refs. [1,4]) for the sake of comparison. All cases correspond to the applied nonlinearity $k=0$ and the RPMs $b(x)$ and $n(x)$ are correctly provided by the system database.

Table 4. Numerical experiments simulating an intruder having access to the PhC-MOEA encryption machine (CPA). Several combinations of altered input images are considered as CPAs. Authentication results are normalized to the positive four-factor verification for the sake of comparison (Fig. 3(c)). All cases correspond to the applied nonlinearity $k=0$, a number of photon-counts $N_{p}=10^{3}$ and $n=8$ bits .

Table 5. Numerical experiments simulating an intruder having access to the PhC-MOEA decryptionauthentication machine (CCA). Several combinations of altered input images are considered as CCAs. Authentication results are normalized to the positive four-factor verification for the sake of comparison (Fig. 3(c)). All cases correspond to the applied nonlinearity $k=0$, a number of photon-counts $N_{p}=10^{3}$ and $n=8$ bits . 


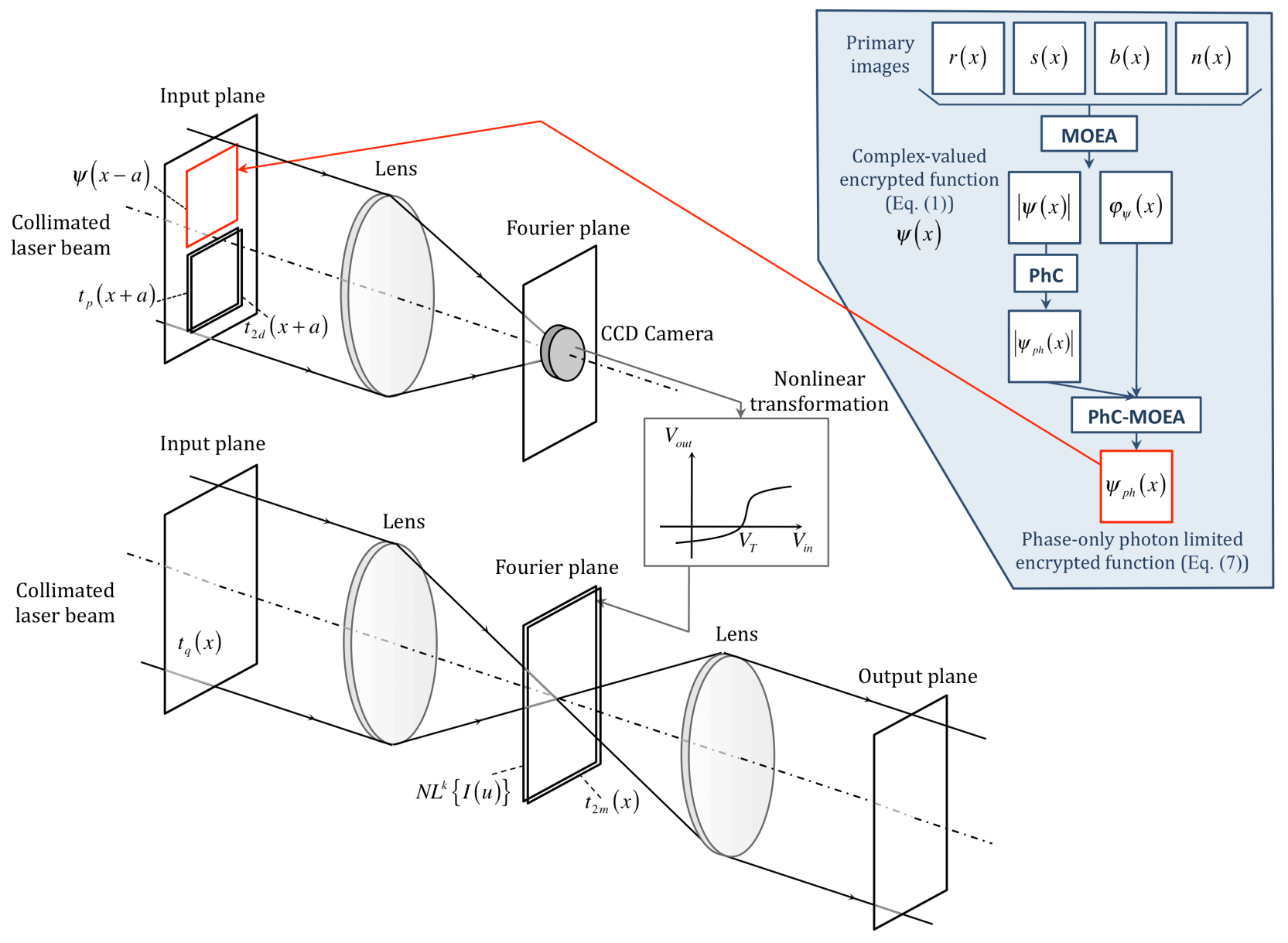

Figure 1 


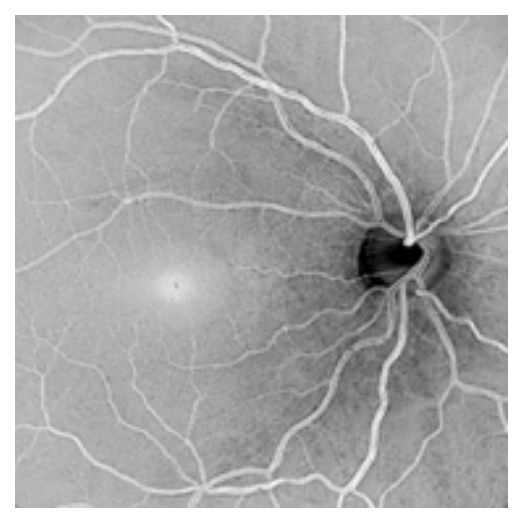

$r(x)$

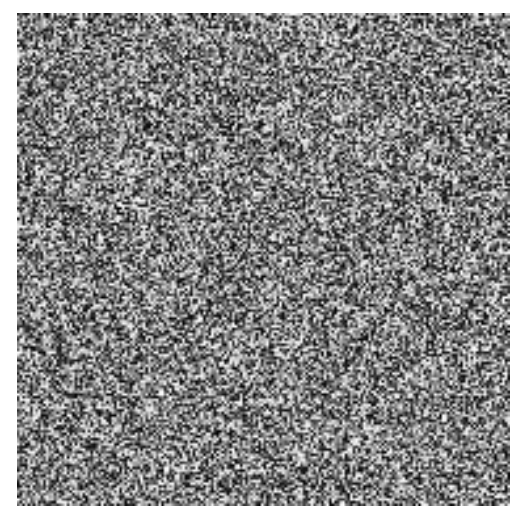

$b(x)$

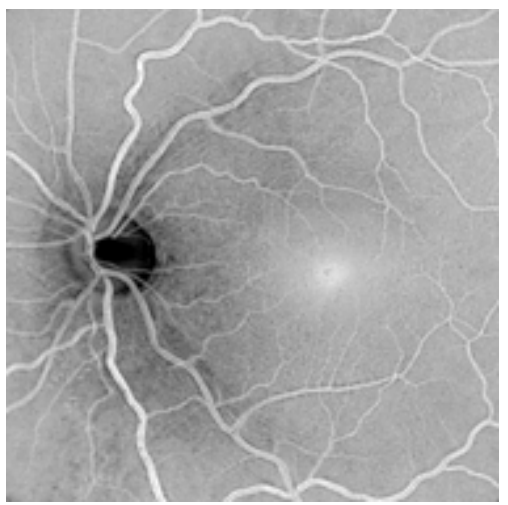

$s(x)$

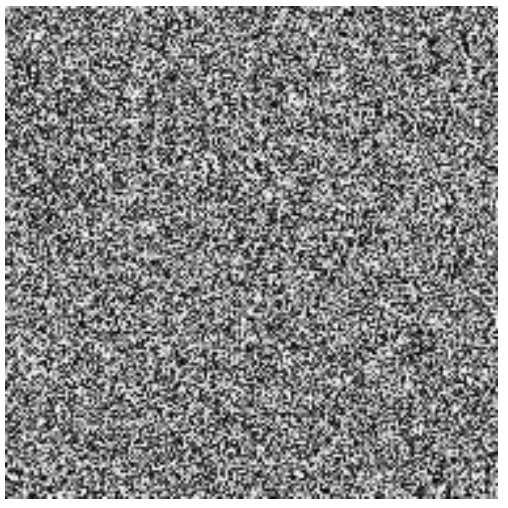

$n(x)$

Figure 2 


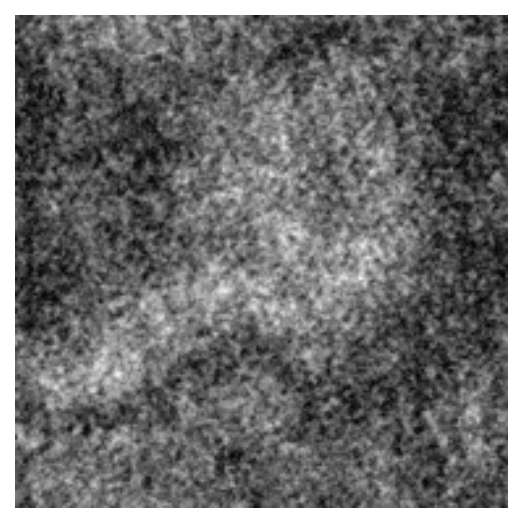

(a)

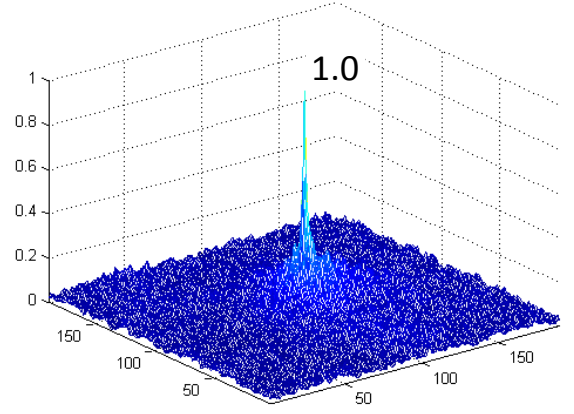

(c)

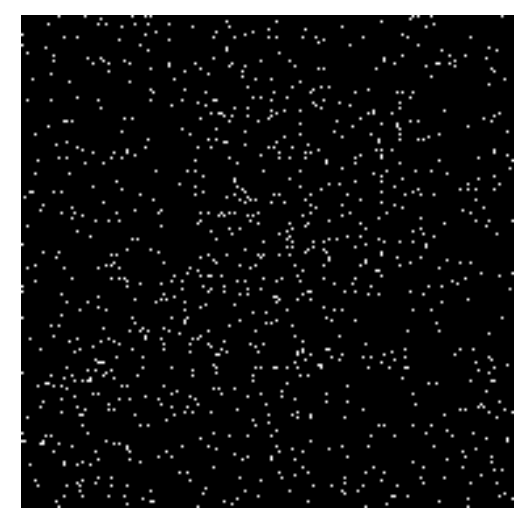

(b)

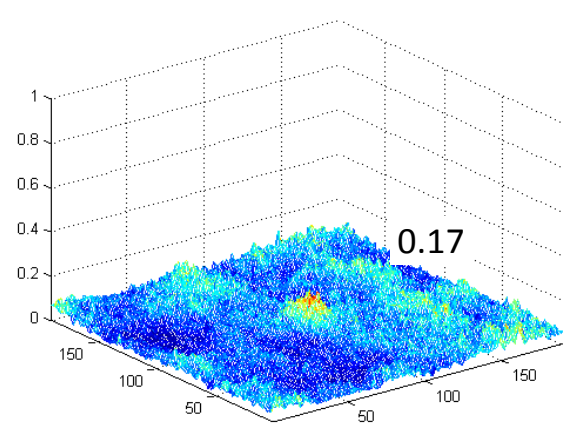

(d)

Figure 3 


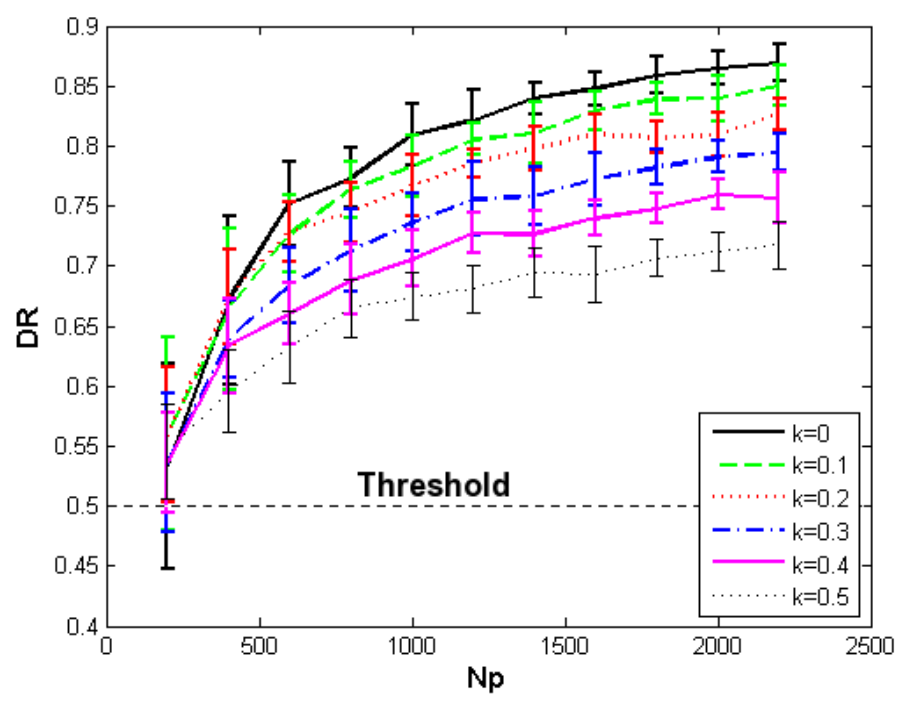

Figure 4 


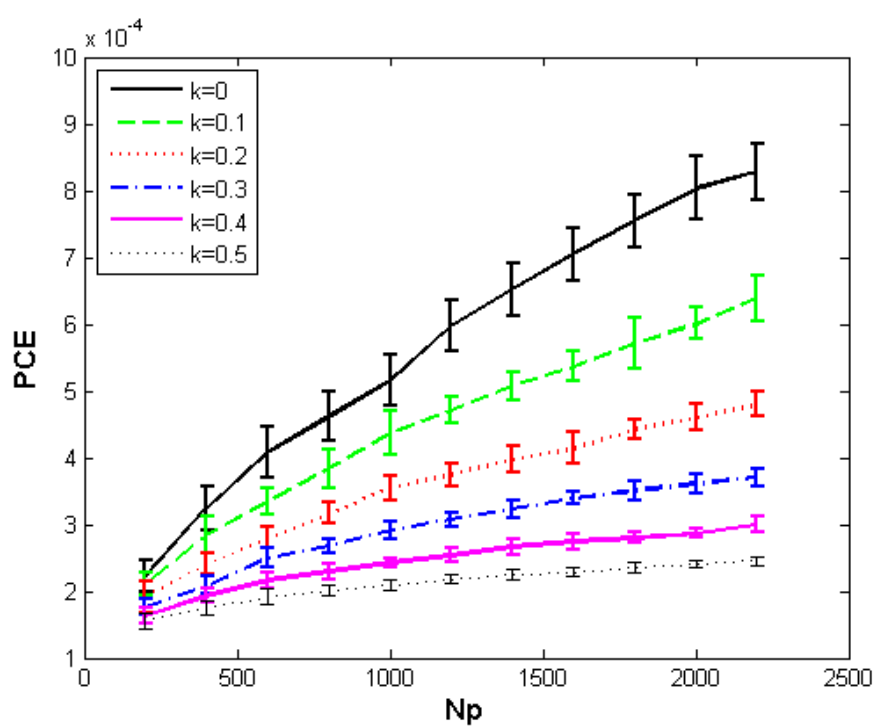

Figure 5 

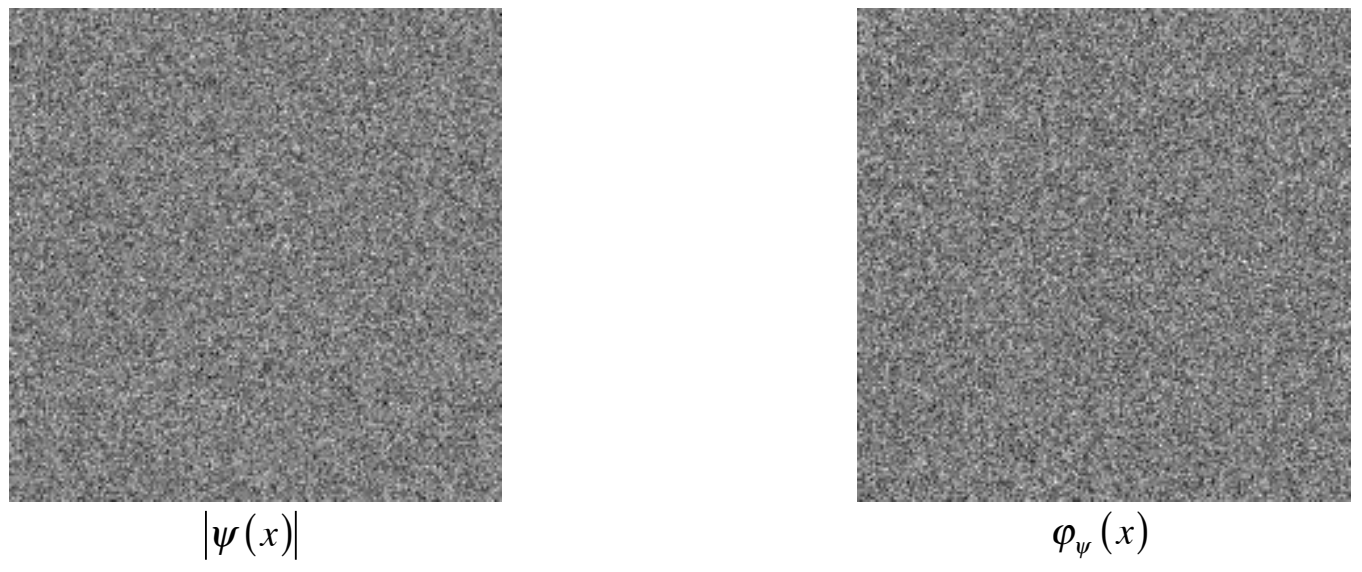

Figure 6 

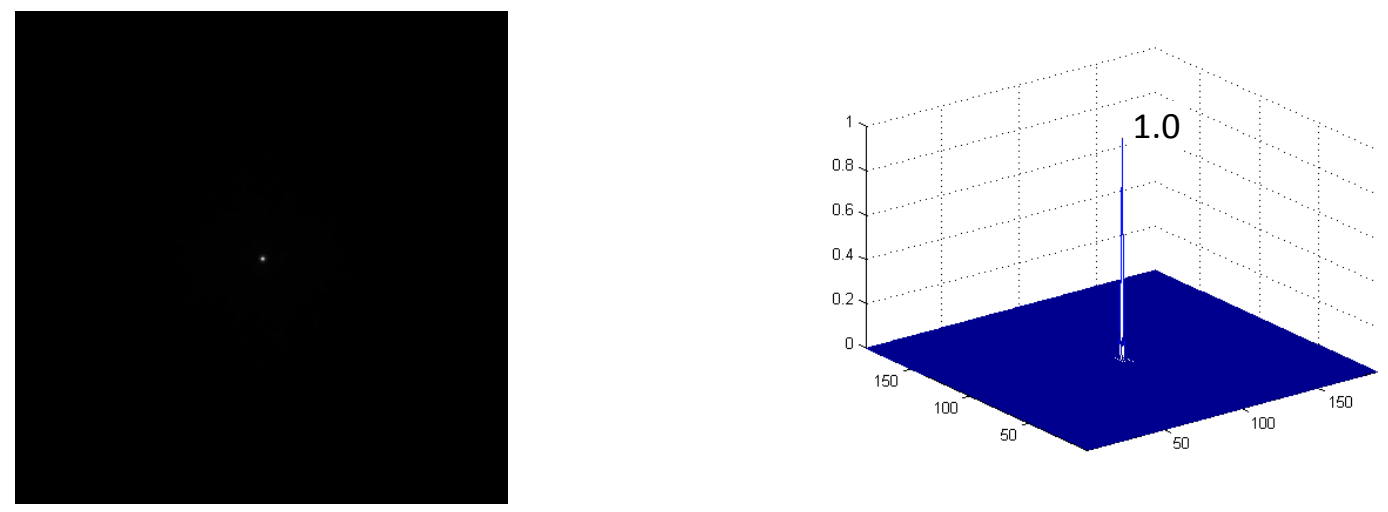

(a)
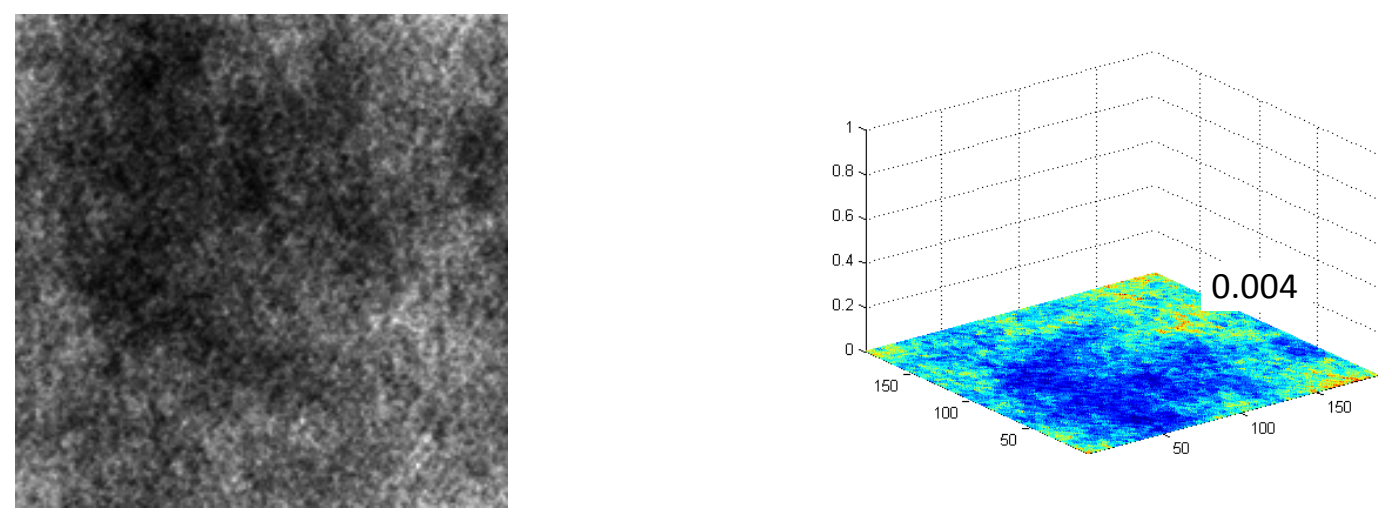

(b)
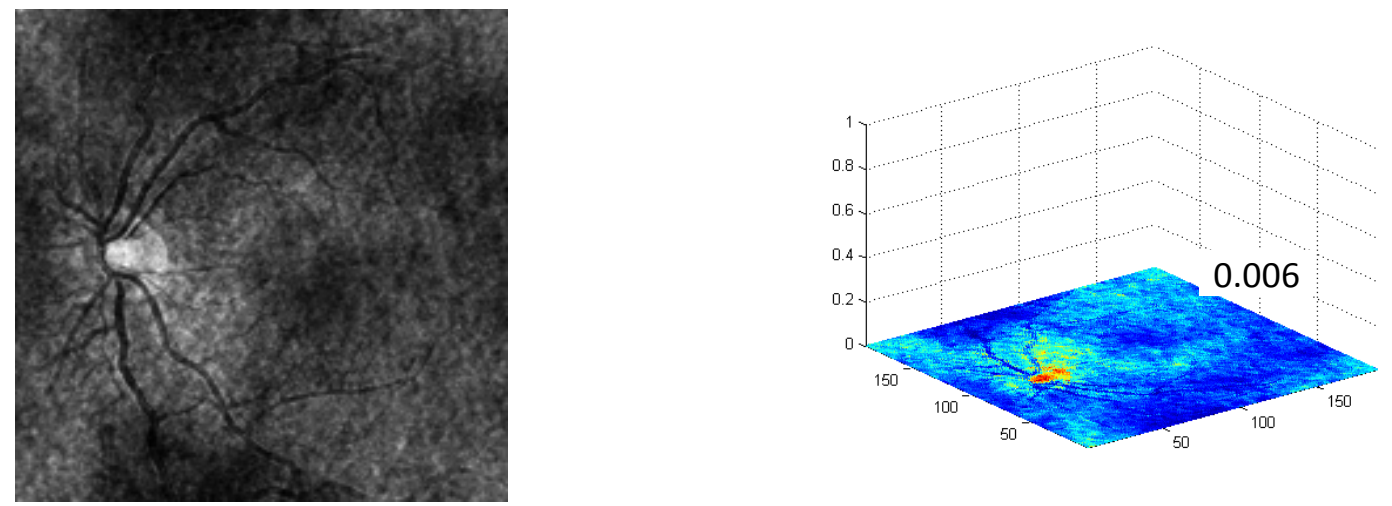

(c)

Figure 7 

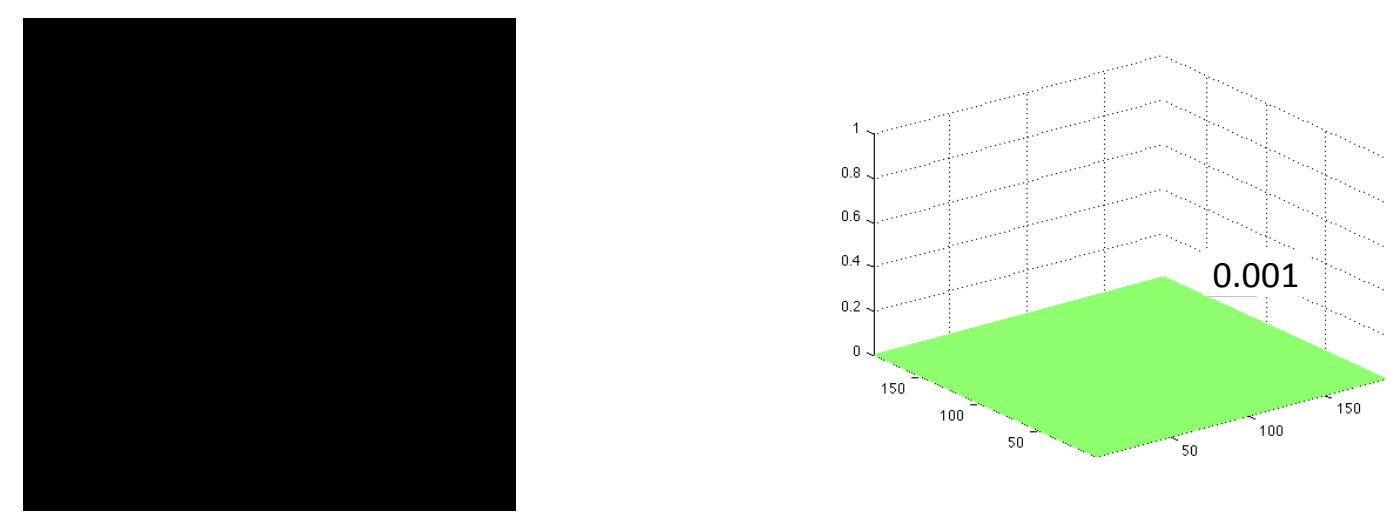

(a)
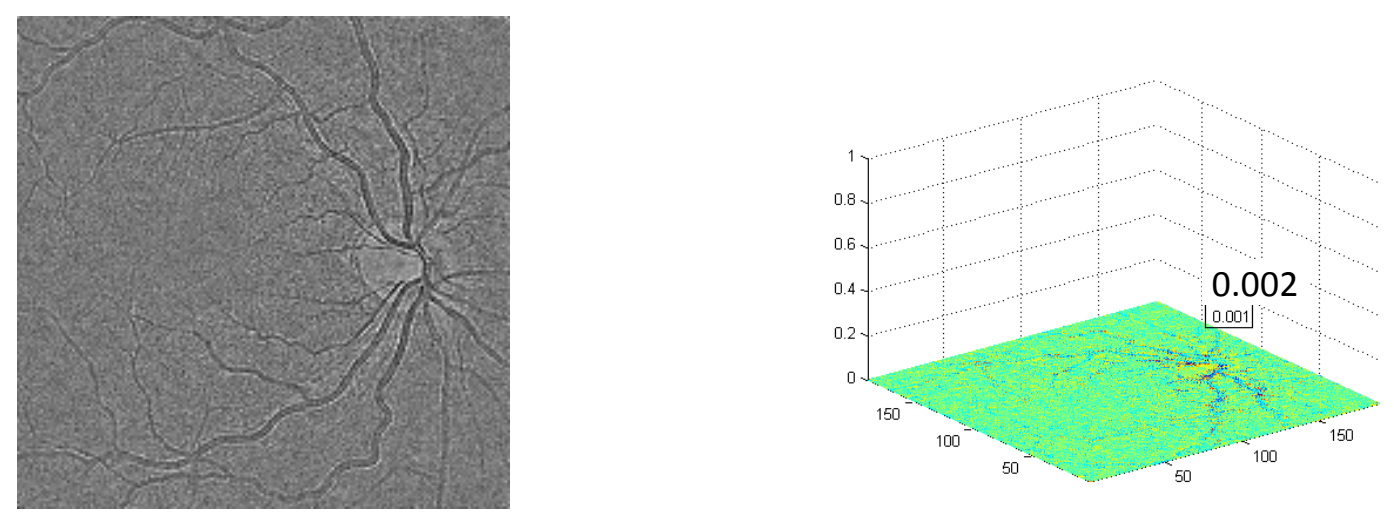

(b)

Figure 8 

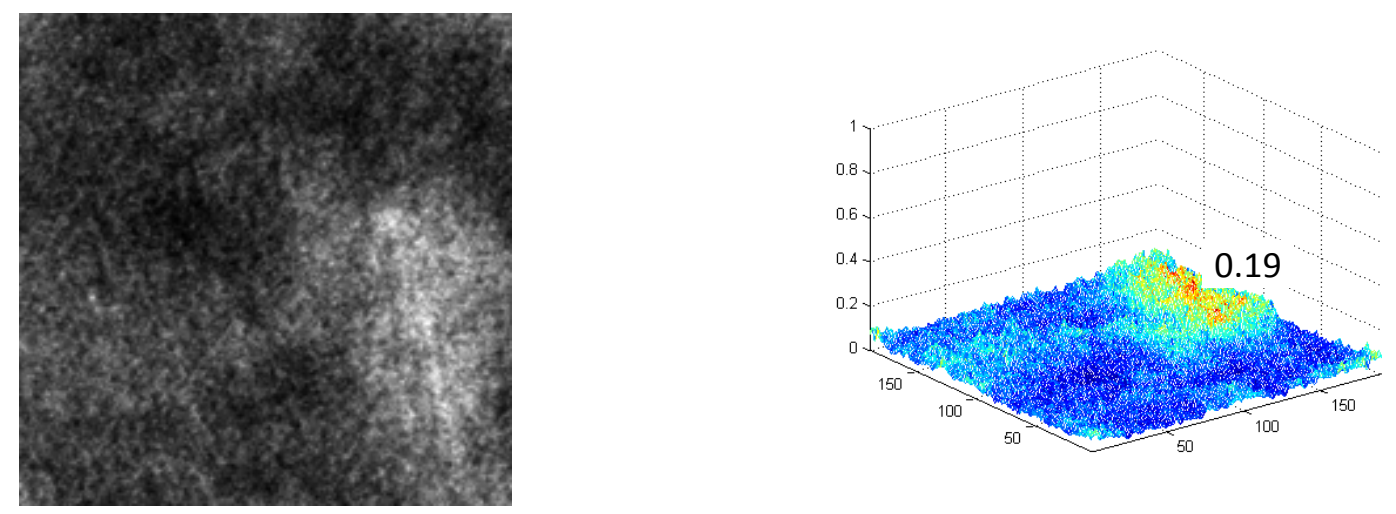

(a)
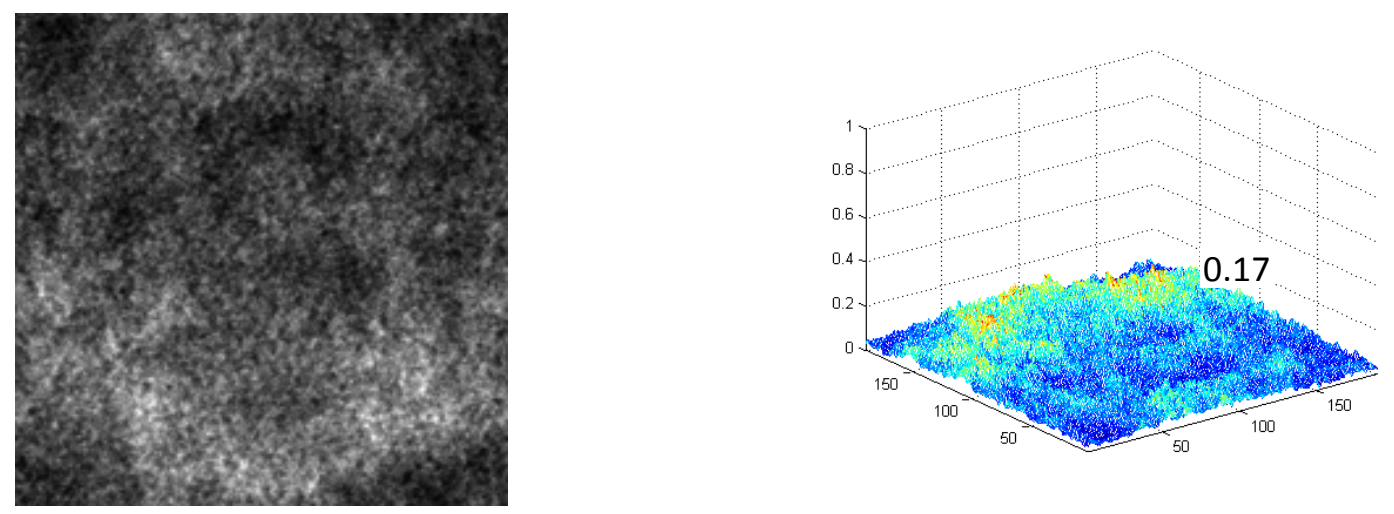

(b)

Figure 9 

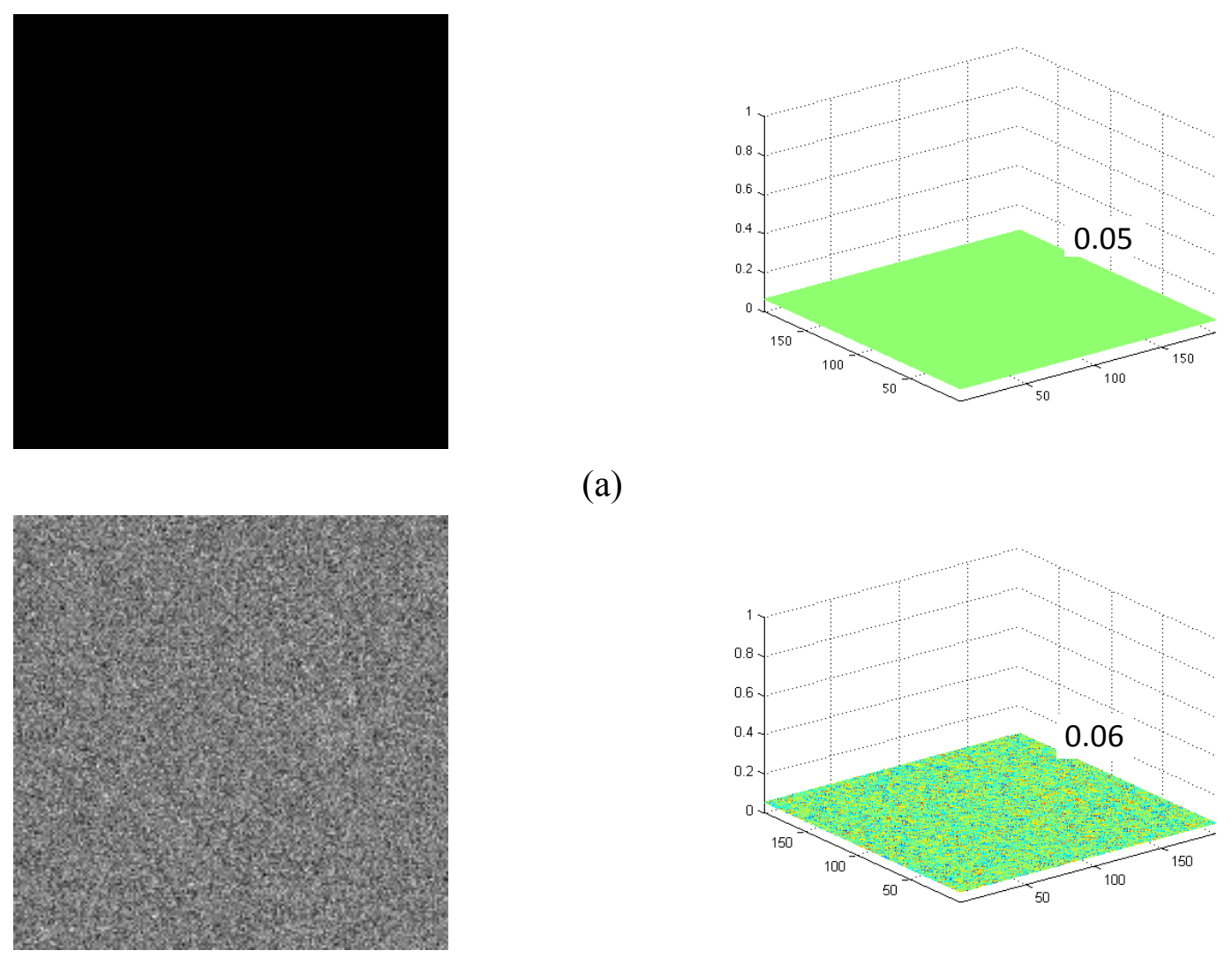

(a)

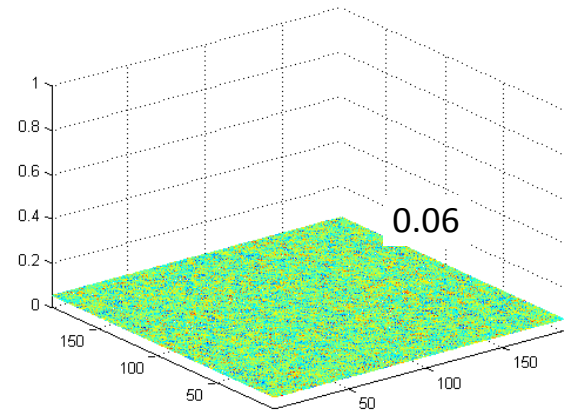

(b)

Figure 10 
Table 1

\begin{tabular}{|c|c|c|c|c|c|}
\hline \multicolumn{7}{|c|}{ PhC-MOEA system } \\
\hline \multicolumn{2}{|c|}{ Retina images } & \multicolumn{2}{c|}{ Phase codes } & & \\
$\boldsymbol{p}(\boldsymbol{x})$ & $\boldsymbol{q}(\boldsymbol{x})$ & $\boldsymbol{m}(\boldsymbol{x})$ & $\boldsymbol{d}(\boldsymbol{x})$ & NMPV* & Authentication Result \\
\hline$p=r$ & $q=s$ & $m=n$ & $d=b$ & 1.0 & Authorized \\
\hline$p \neq r$ & $q \neq s$ & $m=n$ & $d=b$ & 0.17 & Non-Authorized \\
\hline$p=r$ & $q=s$ & $m \neq n$ & $d=b$ & 0.17 & Non-Authorized \\
\hline$p=r$ & $q=s$ & $m=n$ & $d \neq b$ & 0.16 & Non-Authorized \\
\hline$p=r$ & $q=s$ & $m \neq n$ & $d \neq b$ & 0.18 & Non-Authorized \\
\hline
\end{tabular}

$r, s, n$ and $b$ from Fig. 2

*NMPV: Normalized Maximum Peak Value 
Table 2

\begin{tabular}{|c|c|c|c|c|c|}
\hline \multicolumn{5}{|c|}{ MOEA system } \\
\hline $\begin{array}{c}\text { Encryption Stage } \\
\text { (with Chosen-Plaintext Attack) }\end{array}$ & \multicolumn{5}{c|}{ Decryption and Authentication Stage } \\
\hline $\boldsymbol{r}(\boldsymbol{x})$ & $\boldsymbol{s}(\boldsymbol{x})$ & $\boldsymbol{p}(\boldsymbol{x})$ & $\boldsymbol{q}(\boldsymbol{x})$ & NMPV* & Authentication Result \\
\hline$r$ & $s$ & $r$ & $s$ & 1.0 & Authorized \\
\hline 0 & 0 & $r$ & $s$ & 0.0042 & Non-Authorized \\
\hline$d o t$ & 0 & $r$ & $s$ & 0.0044 & Non-Authorized \\
\hline$r$ & 0 & $r$ & $s$ & 0.0042 & Non-Authorized \\
\hline 0 & $d o t$ & $r$ & $s$ & 0.0055 & Non-Authorized \\
\hline$d o t$ & $d o t$ & $r$ & $s$ & 0.0055 & Non-Authorized \\
\hline$r$ & $d o t$ & $r$ & $s$ & 0.0081 & Non-Authorized \\
\hline
\end{tabular}

$r$ and $s$ from Fig. 2

*NMPV: Normalized Maximum Peak Value

Table 3

\begin{tabular}{|c|c|c|c|c|c|}
\hline \multicolumn{5}{|c|}{ MOEA system } \\
\hline \multicolumn{2}{|c|}{ Encryption Stage } & \multicolumn{5}{c|}{$\begin{array}{r}\text { Decryption and Authentication Stage } \\
\text { (with Chosen-Ciphertext Attack) }\end{array}$} \\
\hline$r(\boldsymbol{x})$ & $\boldsymbol{s}(\boldsymbol{x})$ & $\boldsymbol{p}(\boldsymbol{x})$ & $\boldsymbol{q}(\boldsymbol{x})$ & NMPV* & Authentication Result \\
\hline$r$ & $s$ & 0 & 0 & 0.0013 & Non-Authorized \\
\hline$r$ & $s$ & $d o t$ & 0 & 0.0013 & Non-Authorized \\
\hline$r$ & $s$ & $r$ & 0 & 0.0013 & Non-Authorized \\
\hline$r$ & $s$ & 0 & $d o t$ & 0.0015 & Non-Authorized \\
\hline$r$ & $s$ & $d o t$ & $d o t$ & 0.0015 & Non-Authorized \\
\hline$r$ & $s$ & $r$ & $d o t$ & 0.0015 & Non-Authorized \\
\hline
\end{tabular}

$r$ and $s$ from Fig. 2

*NMPV: Normalized Maximum Peak Value 
Table 4

\begin{tabular}{|c|c|c|c|c|c|}
\hline \multicolumn{5}{|c|}{ PhC-MOEA system } \\
\hline $\begin{array}{c}\text { Encryption Stage } \\
\text { (with Chosen-Plaintext Attack) }\end{array}$ & \multicolumn{5}{c|}{ Decryption and Authentication Stage } \\
\hline $\boldsymbol{r}(\boldsymbol{x})$ & $\boldsymbol{s}(\boldsymbol{x})$ & $\boldsymbol{p}(\boldsymbol{x})$ & $\boldsymbol{q}(\boldsymbol{x})$ & NMPV* & Authentication Result \\
\hline 0 & 0 & $r$ & $s$ & 0.17 & Non-Authorized \\
\hline$d o t$ & 0 & $r$ & $s$ & 0.19 & Non-Authorized \\
\hline$r$ & 0 & $r$ & $s$ & 0.16 & Non-Authorized \\
\hline 0 & $d o t$ & $r$ & $s$ & 0.18 & Non-Authorized \\
\hline$d o t$ & $d o t$ & $r$ & $s$ & 0.17 & Non-Authorized \\
\hline$r$ & $d o t$ & $r$ & $s$ & 0.19 & Non-Authorized \\
\hline
\end{tabular}

$r$ and $s$ from Fig. 2

*NMPV: Normalized Maximum Peak Value

Table 5

\begin{tabular}{|c|c|c|c|c|c|}
\hline \multicolumn{5}{|c|}{ PhC-MOEA system } \\
\hline \multicolumn{2}{|c|}{ Encryption Stage } & \multicolumn{5}{c|}{$\begin{array}{r}\text { Decryption and Authentication Stage } \\
\text { (with Chosen-Ciphertext Attack) }\end{array}$} \\
\hline $\boldsymbol{r}(\boldsymbol{x})$ & $\boldsymbol{s}(\boldsymbol{x})$ & $\boldsymbol{p}(\boldsymbol{x})$ & $\boldsymbol{q}(\boldsymbol{x})$ & NMPV* $^{*}$ & Authentication Result \\
\hline$r$ & $s$ & 0 & 0 & 0.052 & Non-Authorized \\
\hline$r$ & $s$ & $d o t$ & 0 & 0.052 & Non-Authorized \\
\hline$r$ & $s$ & $r$ & 0 & 0.052 & Non-Authorized \\
\hline$r$ & $s$ & 0 & $d o t$ & 0.055 & Non-Authorized \\
\hline$r$ & $s$ & $d o t$ & $d o t$ & 0.055 & Non-Authorized \\
\hline$r$ & $s$ & $r$ & $d o t$ & 0.055 & Non-Authorized \\
\hline
\end{tabular}

$r$ and $s$ from Fig. 2

*NMPV: Normalized Maximum Peak Value 